\title{
Lipschitz continuous and compact composition operators in hyperbolic classes
}

\section{Taskinen, Jari}

2011

Taskinen , J , Rättyä , J \& Pérez-González , F 2011 , ' Lipschitz continuous and compact composition operators in hyperbolic classes ' , Mediterranean journal of mathematics, vol. 8 , no. 1 , pp. 123-135 . https://doi.org/10.1007/s00009-010-0054-z

http://hdl.handle.net/10138/26022

https://doi.org/10.1007/s00009-010-0054-z

submittedVersion

Downloaded from Helda, University of Helsinki institutional repository.

This is an electronic reprint of the original article.

This reprint may differ from the original in pagination and typographic detail.

Please cite the original version. 


\title{
Lipschitz continuous and compact composi- tion operators in hyperbolic classes
}

\author{
Fernando Pérez-González, Jouni Rättyä and Jari Taskinen
}

\begin{abstract}
Natural metrics in the hyperbolic $\alpha$-Bloch-, weighted Dirichletand $Q_{p}$-classes are introduced, and these classes are shown to be complete metric spaces with respect to the corresponding metrics. Then Lipschitz continuous and compact composition operators $C_{\varphi}(f)=f \circ \varphi$ acting from the hyperbolic $\alpha$-Bloch-class to the hyperbolic weighted Dirichlet- or $Q_{p}$-class are characterized by conditions depending on the symbol $\varphi$ only.
\end{abstract}

Mathematics Subject Classification (2010). Primary 47B38; Secondary 30D45, 30D50, 46E15.

Keywords. Composition operator, hyperbolic class, Lipschitz continuous, completely continuous, Bloch-space, Dirichlet-space, $Q_{p}$-space.

\section{Introduction}

Hyperbolic function classes are subsets of the class $B(\mathbb{D})$ of all analytic functions $f$ in the unit disc $\mathbb{D}$ such that $|f(z)|<1$ for all $z \in \mathbb{D}$. They are usually defined by using either the hyperbolic derivative $f^{*}(z):=\left|f^{\prime}(z)\right| /\left(1-|f(z)|^{2}\right)$ of $f \in B(\mathbb{D})$, or the hyperbolic distance $\rho(f(z), 0):=\frac{1}{2} \log ((1+|f(z)|) /(1-$ $|f(z)|))$ between $f(z)$ and 0 .

Hyperbolic function classes, like the $\alpha$-Bloch-class of those $f \in B(\mathbb{D})$ for which

$$
\|f\|_{\mathcal{B}_{\alpha}^{*}}:=\sup _{z \in \mathbb{D}} f^{*}(z)\left(1-|z|^{2}\right)^{\alpha}<\infty
$$

are not linear spaces. It is thus slightly surprising that a satisfactory theory of analytic composition operators still exists for these classes. In this paper we introduce natural metrics on the hyperbolic $\alpha$-Bloch-, $Q_{p^{-}}$and Dirichletclasses, and show that these classes are complete metric spaces with respect

The research of the first two authors was partially supported by MICINN-Spain (MTM2005-07347, MTM2006-26267-E, MTM2007-30904-E and MTM2008-05891), and ESF Research Networking Programme HCAA. The second author was in addition partially supported by the Academy of Finland projects 210245 and 121281. 
to the corresponding metrics. The main observation of our paper is that, for a composition operator $C_{\varphi}(f):=f \circ \varphi$ induced by $\varphi \in B(\mathbb{D})$, the Lipschitzcontinuity on hyperbolic classes plays the same role as the continuity or the boundedness on the usual Banach-function spaces. This analogy even extends to the study of compact operators (or completely continuous operators in the language of general nonlinear functional analysis).

Yamashita was probably the first one to consider systematically hyperbolic function classes. He introduced and studied hyperbolic Hardy-, BMOAand Dirichlet-classes in $[7,8,9,10,11,12]$. More recently, Smith studied inner functions in the hyperbolic little Bloch-class [4], and the hyperbolic counterparts of the $Q_{p}$-spaces were studied by $\mathrm{Li}$ [2] and $\mathrm{Li}$ et. al. [3]. Further, hyperbolic classes even arise naturally in the study of composition operators acting on Banach spaces of analytic functions in $\mathbb{D}$. For example, a result by Xiao shows that a composition operator $C_{\varphi}$ mapping from the classical Bloch-space $\mathcal{B}$ to the conventional $\alpha$-Bloch-space $\mathcal{B}_{\alpha}$ is bounded if and only if the symbol $\varphi$ belongs to the hyperbolic $\alpha$-Bloch-class $\mathcal{B}_{\alpha}^{*}[5]$.

The paper is organized as follows. In Section 2 we introduce the hyperbolic $\alpha$-Bloch-, weighted Dirichlet- and $Q_{p}$-classes and natural metrics on them. We also present the main results, the characterizations of Lipschitz continuous and compact composition operators mapping from the hyperbolic $\alpha$-Bloch-class to the hyperbolic weighted Dirichlet- or $Q_{p}$-class by conditions depending on the symbol $\varphi$ only. The proofs of the results are given in Sections $3-6$ in the chronological order. Section 7 is devoted to several remarks on possible choices of metrics.

\section{Basic definitions and results}

We denote by $\mathbb{D}=\{z:|z|<1\}$ the unit disc of the complex plane, and $B(\mathbb{D})$ stands for the class of all analytic functions $f$ in $\mathbb{D}$ for which $|f(z)|<1$ for all $z \in \mathbb{D}$.

If $(X, d)$ is a metric space, we denote the open and closed balls with center $x$ and radius $r>0$ by $B(x, r):=\{y \in X: d(y, x)<r\}$ and $\bar{B}(x, r):=$ $\{y \in X: d(y, x) \leq r\}$, respectively. By $C, c, C_{1}$ and so on we denote strictly positive constants which may vary from place to place but not in the same sequence of inequalities.

Denoting $f^{*}(z)=\left|f^{\prime}(z)\right| /\left(1-|f(z)|^{2}\right)$, the hyperbolic derivative of $f \in$ $B(\mathbb{D})$, the hyperbolic $\alpha$-Bloch-classes $\mathcal{B}_{\alpha}^{*}$ and $\mathcal{B}_{\alpha, 0}^{*}$ are defined as the sets of those $f \in B(\mathbb{D})$ for which

$$
\|f\|_{\mathcal{B}_{\alpha}^{*}}:=\sup _{z \in \mathbb{D}} f^{*}(z)\left(1-|z|^{2}\right)^{\alpha}<\infty
$$

and

$$
\lim _{|z| \rightarrow 1^{-}} f^{*}(z)\left(1-|z|^{2}\right)^{\alpha}=0
$$

respectively. The Schwarz-Pick lemma implies $\mathcal{B}_{\alpha}^{*}=B(\mathbb{D})$ for all $\alpha \geq 1$ with $\|f\|_{\mathcal{B}_{\alpha}^{*}} \leq 1$, and therefore the hyperbolic $\alpha$-Bloch-classes are of interest only 
when $0<\alpha<1$. The usual $\alpha$-Bloch-spaces (defined using the conventional derivative $f^{\prime}$ instead of $f^{*}$ ) and their norms are denoted by the same symbols but without $*$.

It is obvious that $\mathcal{B}_{\alpha}^{*}$ is not a linear space since the sum of two functions in $B(\mathbb{D})$ does not necessarily belong to $B(\mathbb{D})$. However, we can find a natural metric in $\mathcal{B}_{\alpha}^{*}$ by defining

$$
\begin{aligned}
d\left(f, g ; \mathcal{B}_{\alpha}^{*}\right):= & d_{\mathcal{B}_{\alpha}^{*}}(f, g)+\|f-g\|_{\mathcal{B}_{\alpha}}+|f(0)-g(0)| \\
:= & \sup _{z \in \mathbb{D}}\left|\frac{f^{\prime}(z)}{1-|f(z)|^{2}}-\frac{g^{\prime}(z)}{1-|g(z)|^{2}}\right|\left(1-|z|^{2}\right)^{\alpha} \\
& +\|f-g\|_{\mathcal{B}_{\alpha}}+|f(0)-g(0)|
\end{aligned}
$$

for $f, g \in \mathcal{B}_{\alpha}^{*}$. The presence of the conventional $\alpha$-Bloch-norm here is perhaps unexpected. It is motivated by Example 7 below. It shows the phenomenon that, though trivially $d_{\mathcal{B}_{\alpha}^{*}}(f, 0) \geq\|f\|_{\mathcal{B}_{\alpha}}$ for all $f \in \mathcal{B}_{\alpha}^{*}$, the same does no more hold for the differences of two functions: there does not even exist a constant $C>0$ such that

$$
\sup _{z \in \mathbb{D}}\left|\frac{f^{\prime}(z)}{1-|f(z)|^{2}}-\frac{g^{\prime}(z)}{1-|g(z)|^{2}}\right|\left(1-|z|^{2}\right)^{\alpha} \geq C\|f-g\|_{\mathcal{B}_{\alpha}}
$$

would hold for all $f, g \in \mathcal{B}_{\alpha}^{*}, 0<\alpha<1$. At the end of the paper we make some further remarks on the choice of the metric, see Section 7.

Proposition 2.1. The class $\mathcal{B}_{\alpha}^{*}$ equipped with the metric $d\left(\cdot, \cdot ; \mathcal{B}_{\alpha}^{*}\right)$ is a complete metric space. Moreover, $\mathcal{B}_{\alpha, 0}^{*}$ is a closed (and therefore complete) subspace of $\mathcal{B}_{\alpha}^{*}$.

We now turn to consider hyperbolic $Q_{p}$-classes. Recall that, for $0 \leq p<$ $\infty$, the hyperbolic class $Q_{p}^{*}$ consists of those $f \in B(\mathbb{D})$ for which

$$
\|f\|_{Q_{p}^{*}}^{2}:=\sup _{a \in \mathbb{D}} \int_{\mathbb{D}}\left(f^{*}(z)\right)^{2} g^{p}(z, a) d A(z)<\infty,
$$

where $d A$ is the normalized 2-dimensional Lebesgue measure on $\mathbb{D}, g(z, a):=$ $-\log \left|\varphi_{a}(z)\right|$ is the Green's function of $\mathbb{D}$ and $\varphi_{a}(z):=(a-z) /(1-\bar{a} z)$ is the automorphism of $\mathbb{D}$ which interchanges the origin and the point $a \in \mathbb{D}$. Moreover, $f \in Q_{p, 0}^{*}$ if

$$
\lim _{|a| \rightarrow 1^{-}} \int_{\mathbb{D}}\left(f^{*}(z)\right)^{2} g^{p}(z, a) d A(z)=0 .
$$

The Schwarz-Pick lemma implies $Q_{p}^{*}=B(\mathbb{D})$ for all $p>1$, and therefore the hyperbolic $Q_{p}$-classes are considered only when $0 \leq p \leq 1$. For $f, g \in Q_{p}^{*}$, define their distance by

$$
\begin{aligned}
d\left(f, g ; Q_{p}^{*}\right):= & d_{Q_{p}^{*}}(f, g)+\|f-g\|_{Q_{p}}+|f(0)-g(0)| \\
:= & \left(\sup _{a \in \mathbb{D}} \int_{\mathbb{D}}\left|\frac{f^{\prime}(z)}{1-|f(z)|^{2}}-\frac{g^{\prime}(z)}{1-|g(z)|^{2}}\right|^{2} g^{p}(z, a) d A(z)\right)^{\frac{1}{2}} \\
& +\|f-g\|_{Q_{p}}+|f(0)-g(0)| .
\end{aligned}
$$


Proposition 2.2. The class $Q_{p}^{*}$ equipped with the metric $d\left(\cdot, \cdot ; Q_{p}^{*}\right)$ is a complete metric space. Moreover, $Q_{p, 0}^{*}$ is a closed (and therefore complete) subspace of $Q_{p}^{*}$.

According to the definition in [3], a composition operator $C_{\varphi}: \mathcal{B}_{\alpha}^{*} \rightarrow Q_{p}^{*}$ is said to be bounded if there is a positive constant $C$ such that $\left\|C_{\varphi}(f)\right\|_{Q_{p}^{*}} \leq$ $C\|f\|_{\mathcal{B}_{\alpha}^{*}}$ for all $f \in \mathcal{B}_{\alpha}^{*}$. Theorem 2.3 shows that $C_{\varphi}: \mathcal{B}_{\alpha}^{*} \rightarrow Q_{p}^{*}$ is bounded if and only if it is Lipschitz-continuous, that is, if there exists a positive constant $C$ such that

$$
d\left(f \circ \varphi, g \circ \varphi ; Q_{p}^{*}\right) \leq C d\left(f, g ; \mathcal{B}_{\alpha}^{*}\right)
$$

for all $f, g \in \mathcal{B}_{\alpha}^{*}$. By elementary functional analysis, a linear operator between normed spaces is bounded if and only if it is continuous, and the boundedness is trivially also equivalent to the Lipschitz-continuity. So, our result for composition operators in hyperbolic spaces is the correct and natural generalization of the linear operator theory.

Theorem 2.3. Let $0<\alpha \leq 1$ and $0 \leq p \leq 1$. Then the following statements are equivalent:

(1) $C_{\varphi}: \mathcal{B}_{\alpha}^{*} \rightarrow Q_{p}^{*}$ is bounded;

(2) $C_{\varphi}: \mathcal{B}_{\alpha}^{*} \rightarrow Q_{p}^{*}$ is Lipschitz continuous;

(3) $\sup _{a \in \mathbb{D}} \int_{\mathbb{D}} \frac{\left|\varphi^{\prime}(z)\right|^{2}}{\left(1-|\varphi(z)|^{2}\right)^{2 \alpha}} g^{p}(z, a) d A(z)<\infty$.

We now turn to consider compact operators.

Definition 2.4. We call an operator $C_{\varphi}: \mathcal{B}_{\alpha}^{*} \rightarrow Q_{p}^{*}$ compact, if it maps any ball in $\mathcal{B}_{\alpha}^{*}$ onto a precompact set in $Q_{p}^{*}$.

This concept coincides with that of a completely continuous operator in general nonlinear functional analysis (see e.g. [1], Definition 8.1.). However, we prefer to use the term compact operator; recall that the operator is still linear, though the underlying spaces are not.

The following observation is sometimes useful.

Proposition 2.5. If $C_{\varphi}: \mathcal{B}_{\alpha}^{*} \rightarrow Q_{p}^{*}$ is compact, it maps closed balls onto compact sets.

Compactness of composition operators can be characterized in full analogy with the linear case.

Theorem 2.6. Let $0<\alpha \leq 1$ and $0 \leq p \leq 1$. Then the following statements are equivalent:

(1) $C_{\varphi}: \mathcal{B}_{\alpha}^{*} \rightarrow Q_{p}^{*}$ is compact;

(2) $\lim _{r \rightarrow 1^{-}} \sup _{a \in \mathbb{D}} \int_{|\varphi(z)| \geq r} \frac{\left|\varphi^{\prime}(z)\right|^{2}}{\left(1-|\varphi(z)|^{2}\right)^{2 \alpha}} g^{p}(z, a) d A(z)=0$.

Before proving the results presented in this section, we point out an immediate consequence of the proofs of these results. Namely, if $\mathcal{D}_{p}^{*}$ denotes 
the hyperbolic weighted Dirichlet-class which consists of those $f \in B(\mathbb{D})$ for which

$$
\int_{\mathbb{D}}\left(f^{*}(z)\right)^{2}\left(1-|z|^{2}\right)^{p} d A(z)<\infty
$$

then the proof of Proposition 2.2 shows that $\mathcal{D}_{p}^{*}$ is a complete metric space with respect to the metric

$$
\begin{aligned}
d\left(f, g ; \mathcal{D}_{p}^{*}\right):= & d_{\mathcal{D}_{p}^{*}}(f, g)+\|f-g\|_{\mathcal{D}_{p}}+|f(0)-g(0)| \\
:= & \left(\int_{\mathbb{D}}\left|\frac{f^{\prime}(z)}{1-|f(z)|^{2}}-\frac{g^{\prime}(z)}{1-|g(z)|^{2}}\right|^{2}\left(1-|z|^{2}\right)^{p} d A(z)\right)^{\frac{1}{2}} \\
& +\|f-g\|_{\mathcal{D}_{p}}+|f(0)-g(0)| .
\end{aligned}
$$

Moreover, the proofs of Theorems 2.3 and 2.6 yield the following result.

Theorem 2.7. Let $0<\alpha \leq 1$ and $-1<p \leq 1$. Then the following statements are equivalent:

(1) $C_{\varphi}: \mathcal{B}_{\alpha}^{*} \rightarrow \mathcal{D}_{p}^{*}$ is Lipschitz continuous;

(2) $C_{\varphi}: \mathcal{B}_{\alpha}^{*} \rightarrow \mathcal{D}_{p}^{*}$ is compact;

(3) $\int_{\mathbb{D}} \frac{\left|\varphi^{\prime}(z)\right|^{2}}{\left(1-|\varphi(z)|^{2}\right)^{2 \alpha}}\left(1-|z|^{2}\right)^{p} d A(z)<\infty$.

\section{Proof of Proposition 2.1}

For simplicity, denote $d(f, g):=d\left(f, g ; \mathcal{B}_{\alpha}^{*}\right)$. Then clearly $d(f, g) \geq 0, d(f, g)=$ $d(g, f), d(f, h) \leq d(f, g)+d(g, h)$, and $d(f, f)=0$, for all $f, g, h \in \mathcal{B}_{\alpha}^{*}$. It also follows from the presence of the usual $\alpha$-Bloch-term that $d(f, g)=0$ implies $f=g$. Hence, $d$ is a metric on $B_{\alpha}^{*}$.

Let $\left(f_{n}\right)_{n=1}^{\infty}$ be a Cauchy sequence in the metric space $\mathcal{B}_{\alpha}^{*}$, that is, for any $\varepsilon>0$ there is an $N=N(\varepsilon) \in \mathbb{N}$ such that $d\left(f_{n}, f_{m}\right)<\varepsilon$ for all $n, m>N$. Since $\left(f_{n}\right) \subset B(\mathbb{D})$, the family $\left(f_{n}\right)$ is uniformly bounded and hence normal in $\mathbb{D}$. Therefore there exists $f \in B(\mathbb{D})$ and a subsequence $\left(f_{n_{j}}\right)_{j=1}^{\infty}$ such that $f_{n_{j}}$ converges to $f$ uniformly on compact subsets of $\mathbb{D}$. It follows that also $f_{n}$ converges to $f$ uniformly on compact subsets, and by the Cauchy formula, the same also holds for the derivatives. Let $m>N$. Then the uniform convergence yields

$$
\begin{aligned}
& \left|\frac{f^{\prime}(z)}{1-|f(z)|^{2}}-\frac{f_{m}^{\prime}(z)}{1-\left|f_{m}(z)\right|^{2}}\right|\left(1-|z|^{2}\right)^{\alpha} \\
& \quad=\lim _{n \rightarrow \infty}\left|\frac{f_{n}^{\prime}(z)}{1-\left|f_{n}(z)\right|^{2}}-\frac{f_{m}^{\prime}(z)}{1-\left|f_{m}(z)\right|^{2}}\right|\left(1-|z|^{2}\right)^{\alpha} \\
& \quad \leq \lim _{n \rightarrow \infty} d\left(f_{n}, f_{m}\right) \leq \varepsilon
\end{aligned}
$$

for all $z \in \mathbb{D}$, and it follows that $\|f\|_{\mathcal{B}_{\alpha}^{*}} \leq\left\|f_{m}\right\|_{\mathcal{B}_{\alpha}^{*}}+\varepsilon$. Thus $f \in \mathcal{B}_{\alpha}^{*}$ as desired. Moreover, (3.1) and the completeness of the usual $\alpha$-Bloch-space imply that $\left(f_{n}\right)_{n=1}^{\infty}$ converges to $f$ with respect to the metric $d$.

The second part of the assertion follows by (3.1). 


\section{Proof of Proposition 2.2}

As in the previous section we find that $d\left(\cdot, \cdot ; Q_{p}^{*}\right)$ is a metric in $Q_{p}^{*}$. For the completeness proof, let $\left(f_{n}\right)$ be a Cauchy sequence in $Q_{p}^{*}$. As in the proof of Proposition 2.1 we find an $f \in B(\mathbb{D})$ such that $f_{n}$ converges to $f$ uniformly on compact subsets of $\mathbb{D}$. Let $m>N$ and $0<r<1$. Then Fatou's lemma yields

$$
\begin{aligned}
& \int_{\Delta(0, r)}\left|\frac{f^{\prime}(z)}{1-|f(z)|^{2}}-\frac{f_{m}^{\prime}(z)}{1-\left|f_{m}(z)\right|^{2}}\right|^{2} g^{p}(z, a) d A(z) \\
& =\int_{\Delta(0, r)} \lim _{n \rightarrow \infty}\left|\frac{f_{n}^{\prime}(z)}{1-\left|f_{n}(z)\right|^{2}}-\frac{f_{m}^{\prime}(z)}{1-\left|f_{m}(z)\right|^{2}}\right|^{2} g^{p}(z, a) d A(z) \\
& \leq \lim _{n \rightarrow \infty} \int_{\mathbb{D}}\left|\frac{f_{n}^{\prime}(z)}{1-\left|f_{n}(z)\right|^{2}}-\frac{f_{m}^{\prime}(z)}{1-\left|f_{m}(z)\right|^{2}}\right|^{2} g^{p}(z, a) d A(z) \leq \varepsilon^{2},
\end{aligned}
$$

and by letting $r \rightarrow 1^{-}$, it follows that

$$
\int_{\mathbb{D}}\left(f^{*}(z)\right)^{2} g^{p}(z, a) d A(z) \leq 2 \varepsilon^{2}+2 \int_{\mathbb{D}}\left(f_{m}^{*}(z)\right)^{2} g^{p}(z, a) d A(z) .
$$

This yields $\|f\|_{Q_{p}^{*}}^{2} \leq 2 \varepsilon+2\left\|f_{m}\right\|_{Q_{p}^{*}}^{2}$, and thus $f \in Q_{p}^{*}$. We also find that $f_{n} \rightarrow f$ with respect to the metric of $Q_{p}^{*}$.

The second part of the assertion follows by (4.1).

\section{Proof of Theorem 2.3}

It is known that $C_{\varphi}: \mathcal{B}_{\alpha}^{*} \rightarrow Q_{p}^{*}$ is bounded if and only if (3) is satisfied [3]. Therefore it suffices to prove that the assertions (2) and (3) are equivalent.

Assume first that $C_{\varphi}: \mathcal{B}_{\alpha}^{*} \rightarrow Q_{p}^{*}$ is Lipschitz continuous, that is, there exists a positive constant $C$ such that $d\left(f \circ \varphi, g \circ \varphi ; Q_{p}^{*}\right) \leq C d\left(f, g ; \mathcal{B}_{\alpha}^{*}\right)$ for all $f, g \in \mathcal{B}_{\alpha}^{*}$. Taking $g=0$, this implies

$$
\|f \circ \varphi\|_{Q_{p}^{*}} \leq C\left(\|f\|_{\mathcal{B}_{\alpha}^{*}}+\|f\|_{\mathcal{B}_{\alpha}}+|f(0)|\right)
$$

for all $f \in \mathcal{B}_{\alpha}^{*}$. The assertion (3) for $\alpha=1$ follows by choosing $f(z)=z$ in (5.1). If $0<\alpha<1$, then

$$
\begin{aligned}
|f(z)| & =\left|\int_{0}^{z} f^{\prime}(\zeta) d \zeta+f(0)\right| \leq\|f\|_{\mathcal{B}_{\alpha}} \int_{0}^{|z|} \frac{d x}{\left(1-x^{2}\right)^{\alpha}}+|f(0)| \\
& \leq(1-\alpha)^{-1}\|f\|_{\mathcal{B}_{\alpha}}+|f(0)|,
\end{aligned}
$$

and therefore [6, Theorem 2.1.1] implies the existence of $f, g \in \mathcal{B}_{\alpha}^{*}$ such that

$$
\left(\left|f^{\prime}(z)\right|+\left|g^{\prime}(z)\right|\right)\left(1-|z|^{2}\right)^{\alpha} \geq C>0
$$

for all $z \in \mathbb{D}$. Combining (5.1) and (5.3) we obtain

$$
\begin{aligned}
& \left.\|f\|_{\mathcal{B}_{\alpha}^{*}}+\|g\|_{\mathcal{B}_{\alpha}^{*}}+\|f\|_{\mathcal{B}_{\alpha}}+\|g\|_{\mathcal{B}_{\alpha}}+|f(0)|+|g(0)|\right) \\
& \geq C \int_{\mathbb{D}} \frac{\left|\varphi^{\prime}(z)\right|^{2}}{\left(1-|\varphi(z)|^{2}\right)^{2 \alpha}} g^{p}(z, a) d A(z)
\end{aligned}
$$


from which the assertion (3) follows.

Assume now that (3) is satisfied. The inequality (5.2) yields $\mid f(\varphi(0))-$ $g(\varphi(0))\left|\leq(1-\alpha)^{-1}\|f-g\|_{\mathcal{B}_{\alpha}}+\right| f(0)-g(0) \mid$, and therefore

$$
\begin{aligned}
d\left(f \circ \varphi, g \circ \varphi ; Q_{p}^{*}\right)= & d_{Q_{p}^{*}}(f \circ \varphi, g \circ \varphi)+\|f \circ \varphi-g \circ \varphi\|_{Q_{p}} \\
& +|f(\varphi(0))-g(\varphi(0))| \\
\leq & d_{\mathcal{B}_{\alpha}^{*}}(f, g)\left(\sup _{a \in \mathbb{D}} \int_{\mathbb{D}} \frac{\left|\varphi^{\prime}(z)\right|^{2}}{\left(1-|\varphi(z)|^{2}\right)^{2 \alpha}} g^{p}(z, a) d A(z)\right)^{\frac{1}{2}} \\
& +\|f-g\|_{\mathcal{B}_{\alpha}}\left(\sup _{a \in \mathbb{D}} \int_{\mathbb{D}} \frac{\left|\varphi^{\prime}(z)\right|^{2}}{\left(1-|\varphi(z)|^{2}\right)^{2 \alpha}} g^{p}(z, a) d A(z)\right)^{\frac{1}{2}} \\
& +\|f-g\|_{\mathcal{B}_{\alpha}}(1-\alpha)^{-1}+|f(0)-g(0)| \\
\leq & C^{\prime} d\left(f, g ; \mathcal{B}_{\alpha}^{*}\right) .
\end{aligned}
$$

Thus $C_{\varphi}: \mathcal{B}_{\alpha}^{*} \rightarrow Q_{p}^{*}$ is Lipschitz continuous, if (3) is satisfied.

\section{Proofs of Proposition 2.5 and Theorem 2.6}

As regards to Proposition 2.5, if $B \subset \mathcal{B}_{\alpha}^{*}$ is a closed ball and $g \in Q_{p}^{*}$ belongs to the closure of $C_{\varphi}(B)$, we can find a sequence $\left(f_{n}\right)_{n=1}^{\infty} \subset B$ such that $f_{n} \circ \varphi \rightarrow g$ in $Q_{p}^{*}$ as $n \rightarrow \infty$. But $\left(f_{n}\right)_{n=1}^{\infty}$ is a normal family, hence it has a subsequence $\left(f_{n_{j}}\right)_{j=1}^{\infty}$ converging uniformly on the compact subsets of $\mathbb{D}$ to an analytic function $f$. As in earlier arguments (see (3.1)) we get a pointwise estimate which shows that $f$ must belong to the closed ball $B$. On the other hand, also the sequence $\left(f_{n_{j}} \circ \varphi\right)_{j=1}^{\infty}$ converges uniformly on compacta to an analytic function, which is $g$. We get $g=f \circ \varphi$, i.e. $g$ belongs to $C_{\varphi}(B)$. Thus, this set is closed and also compact.

Let us turn to Theorem 2.6. We first assume that (2) holds. Let $B:=$ $\bar{B}(g, \varrho) \subset \mathcal{B}_{\alpha}^{*}$, where $g \in B_{\alpha}^{*}$ and $\varrho>0$, be a closed ball, and let $\left(f_{n}\right)_{n=1}^{\infty} \subset B$ be any sequence. We show that its image has a convergent subsequence in $Q_{p}^{*}$, which proves the compactness of $C_{\varphi}$ by definition.

Again, $\left(f_{n}\right)_{n=1}^{\infty} \subset B(\mathbb{D})$ is a normal family, hence, there is a subsequence $\left(f_{n_{j}}\right)_{j=1}^{\infty}$ which converges uniformly on compact subsets to an analytic function $f$. By the Cauchy formula for the derivative of an analytic function, also the sequence $\left(f_{n_{j}}^{\prime}\right)_{j=1}^{\infty}$ converges uniformly on compacta to $f^{\prime}$. It follows that also the sequences $\left(f_{n_{j}} \circ \varphi\right)_{j=1}^{\infty}$ and $\left(f_{n_{j}}^{\prime} \circ \varphi\right)_{j=1}^{\infty}$ converge uniformly on compact subsets of $\mathbb{D}$ to $f \circ \varphi$ and $f^{\prime} \circ \varphi$, respectively. Moreover, $f \in B \subset \mathcal{B}_{\alpha}^{*}$ 
since for any fixed $R, 0<R<1$, the uniform convergence yields

$$
\begin{aligned}
& \sup _{|z| \leq R}\left|\frac{f^{\prime}(z)}{1-|f(z)|^{2}}-\frac{g^{\prime}(z)}{1-|g(z)|^{2}}\right|\left(1-|z|^{2}\right)^{\alpha} \\
& \quad+\sup _{|z| \leq R}\left|f^{\prime}(z)-g^{\prime}(z)\right|\left(1-|z|^{2}\right)^{\alpha}+|f(0)-g(0)| \\
& =\lim _{j \rightarrow \infty}\left(\sup _{|z| \leq R}\left|\frac{f_{n_{j}}^{\prime}(z)}{1-\left|f_{n_{j}}(z)\right|^{2}}-\frac{g^{\prime}(z)}{1-|g(z)|^{2}}\right|\left(1-|z|^{2}\right)^{\alpha}\right. \\
& \left.\quad+\sup _{|z| \leq R}\left|f_{n_{j}}^{\prime}(z)-g^{\prime}(z)\right|\left(1-|z|^{2}\right)^{\alpha}+|f(0)-g(0)|\right) \leq \varrho,
\end{aligned}
$$

hence, $d\left(f, g ; \mathcal{B}_{\alpha}^{*}\right) \leq \varrho$.

Let $\varepsilon>0$. Since (2) is satisfied, we may fix $r, 0<r<1$, such that

$$
\sup _{a \in \mathbb{D}} \int_{|\varphi(z)| \geq r} \frac{\left|\varphi^{\prime}(z)\right|^{2}}{\left(1-|\varphi(z)|^{2}\right)^{2 \alpha}} g^{p}(z, a) d A(z)<\varepsilon .
$$

By the uniform convergence, we may fix $N_{1} \in \mathbb{N}$ such that

$$
\left|f_{n_{j}} \circ \varphi(0)-f \circ \varphi(0)\right| \leq \varepsilon
$$

for all $j \geq N_{1}$.

The condition (2) is known to imply the compactness of $C_{\varphi}: \mathcal{B}_{\alpha} \rightarrow Q_{p}$, hence, possibly to passing once more to a subsequence and adjusting the notations, we may assume that

$$
\left\|f_{n_{j}} \circ \varphi-f \circ \varphi\right\|_{Q_{p}} \leq \varepsilon
$$

for $j \geq N_{2}$, for some $N_{2} \in \mathbb{N}$.

Since $\left(f_{n_{j}}\right)_{j=1}^{\infty} \subset B$ and $f \in B$, it follows that

$$
\begin{aligned}
& \sup _{a \in \mathbb{D}} \int_{|\varphi| \geq r}\left|\frac{\left(f_{n_{j}}\right)^{\prime}(\varphi(z))}{1-\left|f_{n_{j}}(\varphi(z))\right|^{2}}-\frac{f^{\prime}(\varphi(z))}{1-|f(\varphi(z))|^{2}}\right|^{2}\left|\varphi^{\prime}(z)\right|^{2} g^{p}(z, a) d A(z) \\
& \leq d_{\mathcal{B}_{\alpha}^{*}}\left(f_{n_{j}}, f\right) \sup _{a \in \mathbb{D}} \int_{|\varphi| \geq r} \frac{\left|\varphi^{\prime}(z)\right|^{2}}{\left(1-|\varphi(z)|^{2}\right)^{2 \alpha}} g^{p}(z, a) d A(z) \\
& \leq C \varepsilon
\end{aligned}
$$

for $C=2 \varrho$. On the other hand, by the uniform convergence on compacta of $\mathbb{D}$, we can find an $N_{3} \in \mathbb{N}$ such that for all $j \geq N_{3}$,

$$
\left|\frac{\left(f_{n_{j}}\right)^{\prime}(\varphi(z))}{1-\left|f_{n_{j}}(\varphi(z))\right|^{2}}-\frac{f^{\prime}(\varphi(z))}{1-|f(\varphi(z))|^{2}}\right| \leq \varepsilon
$$

for all $z$ with $|\varphi(z)| \leq r$, hence, for such $j$,

$$
\begin{aligned}
& \sup _{a \in \mathbb{D}} \int_{|\varphi| \leq r}\left|\frac{\left(f_{n_{j}}\right)^{\prime}(\varphi(z))}{1-\left|f_{n_{j}}(\varphi(z))\right|^{2}}-\frac{f^{\prime}(\varphi(z))}{1-|f(\varphi(z))|^{2}}\right|^{2}\left|\varphi^{\prime}(z)\right|^{2} g^{p}(z, a) d A(z) \\
& \leq \varepsilon\left(\sup _{a \in \mathbb{D}} \int_{|\varphi| \leq r} \frac{\left|\varphi^{\prime}(z)\right|^{2}}{\left(1-|\varphi(z)|^{2}\right)^{2 \alpha}} g^{p}(z, a) d A(z)\right)^{\frac{1}{2}} \leq C \varepsilon,
\end{aligned}
$$


where $C$ is the bound obtained from (3) of Theorem 2.3. Combining (6.1), (6.2), (6.3) and (6.4) we deduce that $f_{n_{j}} \rightarrow f$ in $Q_{p}^{*}$.

As for the converse direction, let $f_{n}(z):=n^{\alpha-1} z^{n} / 2$ for all $n \in N$, $n \geq 2$. Then

$$
\begin{aligned}
\left\|f_{n}\right\|_{\mathcal{B}_{\alpha}^{*}} & =\sup _{z \in \mathbb{D}} \frac{n^{\alpha}|z|^{n-1}}{1-2^{-2 n} n^{2(\alpha-1)}|z|^{2 n}}\left(1-|z|^{2}\right)^{\alpha} \\
& \leq 3 \sup _{z \in \mathbb{D}} n^{\alpha}|z|^{n-1}(1-|z|)^{\alpha} .
\end{aligned}
$$

The function $r^{n-1}(1-r)^{\alpha}$ attains it's maximum at the point $r=1-\alpha /(\alpha+$ $n-1)$, and hence (6.5) has the upper bound

$$
3 n^{\alpha}\left(1-\frac{\alpha}{\alpha+n-1}\right)^{n-1} \cdot\left(\frac{\alpha}{\alpha+n-1}\right)^{\alpha} \leq 3 .
$$

As a consequence, the sequence $\left(f_{n}\right)_{n=1}^{\infty}$ belongs to the ball $\bar{B}(0,3) \subset \mathcal{B}_{\alpha}^{*}$.

We are assuming that $C_{\varphi}$ maps the closed ball $\bar{B}(0,3) \subset \mathcal{B}_{\alpha}^{*}$ into a compact subset of $Q_{p}^{*}$, hence, there exists an unbounded increasing subsequence $\left(n_{j}\right)_{j=1}^{\infty}$ such that the image subsequence $\left(C_{\varphi} f_{n_{j}}\right)_{j=1}^{\infty}$ converges with respect to the norm. Since, both $\left(f_{n}\right)_{n=1}^{\infty}$ and $\left(C_{\varphi} f_{n_{j}}\right)_{j=1}^{\infty}$ converge to the zero function uniformly on compact subsets of $\mathbb{D}$, the limit of the latter sequence must be 0 . Hence,

$$
\left\|n_{j}^{\alpha-1} \varphi^{n_{j}}\right\|_{Q_{p}^{*}} \rightarrow 0
$$

as $j \rightarrow \infty$.

Let $r_{j}:=1-1 / n_{j}$. For all numbers $a, r_{j} \leq a<1$, we have the estimate

$$
\begin{aligned}
\frac{n_{j}^{\alpha} a^{n_{j}-1}}{1-a^{n_{j}}} & =\frac{n_{j}^{\alpha} a^{n_{j}-1}}{(1-a) \sum_{k=0}^{n_{j}-1} a^{k} \geq \frac{n_{j}^{\alpha} a^{n_{j}-1}}{(1-a) n_{j}}} \\
& \geq \frac{n_{j}^{\alpha-1}\left(1-1 / n_{j}\right)^{n_{j}-1}}{1-a} \geq \frac{1}{e} \frac{1}{(1-a)^{\alpha}} .
\end{aligned}
$$

Using (6.7) we obtain

$$
\begin{aligned}
\left\|n_{j}^{\alpha-1} \varphi^{n_{j}}\right\|_{Q_{p}^{*}}^{2} & \geq \sup _{a \in \mathbb{D}} \int_{|\varphi| \geq r_{j}}\left|\frac{n_{j}^{\alpha} \varphi(z)^{n_{j}-1}}{1-\left|\varphi(z)^{n_{j}}\right|^{2}}\right|^{2}\left|\varphi^{\prime}(z)\right|^{2} g^{p}(z, a) d A(z) \\
& \geq \sup _{a \in \mathbb{D}} \frac{1}{4 e^{2}} \int_{|\varphi| \geq r_{j}} \frac{\left|\varphi^{\prime}(z)\right|^{2}}{\left(1-|\varphi(z)|^{2}\right)^{2 \alpha}} g^{p}(z, a) d A(z),
\end{aligned}
$$

and the condition (2) follows by combining (6.6) and (6.8).

\section{Remarks on the choice of the metrics}

There is a good motivation to include the conventional $\alpha$-Bloch-norm in the definition of the metric in $\mathcal{B}_{\alpha}^{*}$. Although clearly $\|f\|_{\mathcal{B}_{\alpha}^{*}} \geq\|f\|_{\mathcal{B}_{\alpha}}$ for all $f \in \mathcal{B}_{\alpha}^{*}$, 
the same does no more hold for the differences of two functions: there does not even exist a constant $C>0$ such that

$$
\sup _{z \in \mathbb{D}}\left|\frac{f^{\prime}(z)}{1-|f(z)|^{2}}-\frac{g^{\prime}(z)}{1-|g(z)|^{2}}\right|\left(1-|z|^{2}\right)^{\alpha} \geq C\|f-g\|_{\mathcal{B}_{\alpha}}
$$

would hold for all $f, g \in \mathcal{B}_{\alpha}^{*}, 0<\alpha<1$, as Example 7 shows. Before going to that we make some related remarks.

Remarks. 1. The term $\|f-g\|_{\mathcal{B}_{\alpha}}$ of the metric is explicitely needed in the proof of Theorem 2.3, see (5.2).

The statement of Theorem 2.3 would hold true, if the metric $d\left(\cdot, \cdot ; \mathcal{B}_{\alpha}^{*}\right)$ of $\mathcal{B}_{\alpha}^{*}$ were replaced by the semimetric $d_{\mathcal{B}_{\alpha}^{*}}$ and if the same were done in the space $Q_{p}^{*}$. Of course, this would have the drawback that the spaces would no more be Hausdorff.

2. In view of Example 7 , the identity mapping $\mathcal{B}_{\alpha}^{*} \rightarrow \mathcal{B}_{\alpha}$ would not be Lipschitz-continuous, unless we included $\|f-g\|_{\mathcal{B}_{\alpha}}$ in (2.1). This would not be satisfactory, since the very basic idea here is to show that Lipschitzcontinuity is the correct generalization of the concept of continuity from the Banach spaces to hyperbolic classes, at least in the study of composition operators.

3. We do not know, if the metric $d\left(f, g ; \mathcal{B}_{\alpha}^{*}\right)$ is equivalent to the metric $d^{*}(f, g):=d_{\mathcal{B}_{\alpha}^{*}}(f, g)+|f(0)-g(0)|$ on the class $\mathcal{B}_{\alpha}^{*}$, i.e. if these metric spaces have the same open and closed sets.

We do not know, if the identity mapping $\left(\mathcal{B}_{\alpha}^{*}, d^{*}\right) \rightarrow \mathcal{B}_{\alpha}$ is still continuous.

By the way, to see that $d^{*}$ is indeed a metric on $\mathcal{B}_{\alpha}^{*}$, the only non-trivial step is to prove that $d^{*}(f, g)=0$ implies $f=g$ in $\mathbb{D}$. If $d^{*}(f, g)=0$, then $f(0)=g(0)$ and

$$
\frac{f^{\prime}(z)}{1-|f(z)|^{2}}=\frac{g^{\prime}(z)}{1-|g(z)|^{2}}
$$

for all $z \in \mathbb{D}$, in particular $f^{\prime}(0)=g^{\prime}(0)$. If $f^{\prime}$ has no zeros (and therefore $g^{\prime}$ neither), then

$$
\frac{f^{\prime}(z)}{g^{\prime}(z)}=\frac{1-|f(z)|^{2}}{1-|g(z)|^{2}} \in \mathbb{R}^{+}
$$

for all $z \in \mathbb{D}$, and it follows that $f=C_{1} g+C_{2}$, where $C_{1} \in \mathbb{R}^{+}$and $C_{2} \in \mathbb{C}$. But $f^{\prime}(0)=g^{\prime}(0)$ yields $C_{1}=1$ and then $f(0)=g(0)$ ensures that $C_{2}=0$, so $f(z)=g(z)$ for all $z \in \mathbb{D}$. If $f^{\prime}$ has a zero of order $m$ in a point $a \in \mathbb{D}$, then also $g^{\prime}$ must have a zero of the same order $m$ in $a \in \mathbb{D}$, and it follows that $f^{\prime} / g^{\prime}$ is positive and real in some neighborhood of $a$. One again deduces $f=C_{1} g+C_{2}$, where $C_{1} \in \mathbb{R}^{+}$and $C_{2} \in \mathbb{C}$, and it follows that $f=g$ in $\mathbb{D}$.

4. There are related open questions for the $Q_{p}^{*}$ and Dirichlet-classes. The above results hold true, if the term $\|f-g\|_{Q_{p}}$ is dropped out from the definition of the metric of $Q_{p}^{*}$ and that of the Dirichlet-classes. We prefer to keep the terms, for aesthetic reasons if nothing else. See also the remark 2 above. 
Example. Pick up the positive numbers $a_{k}$ and $b_{k}, k=1,2$, such that $a_{k} \neq b_{k}$,

$$
\frac{a_{2}}{1-a_{1}^{2}}=\frac{b_{2}}{1-b_{1}^{2}} \quad \text { and } \quad a_{1}+3 a_{2}<1, \quad b_{1}+3 b_{2}<1 .
$$

For example, let $a_{1}=1 / 10=a_{2}, b_{1}=1 / 2$ and $b_{2}=5 / 66$. Define, for all $j \in \mathbb{N}$,

$$
f_{j}(z):=a_{1}+a_{2}\left(1-z+j^{-1}\right)^{\beta}, \quad g_{j}(z):=b_{1}+b_{2}\left(1-z+j^{-1}\right)^{\beta},
$$

where $\beta$ is chosen such that $0<(1-\alpha) / 2<\beta<1-\alpha<1$. For all $j$, these functions belong to $\mathcal{B}_{\alpha, 0}^{*}$. Now

$$
\begin{aligned}
\left\|f_{j}-g_{j}\right\|_{\mathcal{B}_{\alpha}} & =\sup _{z \in \mathbb{D}}\left|f_{j}^{\prime}(z)-g_{j}^{\prime}(z)\right|\left(1-|z|^{2}\right)^{\alpha} \\
& =\sup _{z \in \mathbb{D}}\left(a_{2}-b_{2}\right) \beta\left|1-z+j^{-1}\right|^{\beta-1}\left(1-|z|^{2}\right)^{\alpha},
\end{aligned}
$$

and this approaches to $+\infty$ as $j \rightarrow \infty$, since $a_{2} \neq b_{2}$ and $\beta-1+\alpha<0$.

On the other hand, the functions $f_{j}$ and $g_{j}$ are analytic in the closed unit disc, and therefore the asymptotic relation

$$
\left|\frac{f_{j}^{\prime}(1)}{1-\left|f_{j}(1)\right|^{2}}-\frac{g_{j}^{\prime}(1)}{1-\left|g_{j}(1)\right|^{2}}\right|=\mathcal{O}\left(\frac{1}{j^{2 \beta-1}}\right), \quad j \rightarrow \infty,
$$

can be verified by a direct calculation. If $0<\alpha<\frac{1}{2}$, then we may choose $\beta$ such that $2 \beta-1>0$, and it follows that $d_{\mathcal{B}_{\alpha}^{*}}\left(f_{j}, g_{j}\right)$ is uniformly bounded for all $j$. The general case $0<\alpha<1$ can be proved by applying

$$
\frac{c_{1} \varepsilon^{\beta-1}}{1-c_{2}+c_{3} \varepsilon^{\beta}}=\frac{c_{1} \varepsilon^{\beta-1}}{1-c_{2}}+\mathcal{O}\left(\varepsilon^{2 \beta-1}\right),
$$

valid for small $\varepsilon$. This together with (7.1) yield

$$
\begin{aligned}
\mid & \left|\frac{f_{j}^{\prime}(z)}{1-\left|f_{j}(z)\right|^{2}}-\frac{g_{j}^{\prime}(z)}{1-\left|g_{j}(z)\right|^{2}}\right| \\
= & \mid \frac{a_{2} \beta\left(1-z+j^{-1}\right)^{\beta-1}}{1-\left(a_{1}^{2}+2 a_{1} a_{2}\left(1-z+j^{-1}\right)^{\beta}+a_{2}^{2}\left(1-z+j^{-1}\right)^{2 \beta}\right)} \\
& -\frac{b_{2} \beta\left(1-z+j^{-1}\right)^{\beta-1}}{1-\left(b_{1}^{2}+2 b_{1} b_{2}\left(1-z+j^{-1}\right)^{\beta}+b_{2}^{2}\left(1-z+j^{-1}\right)^{2 \beta}\right)} \mid \\
= & \mid \frac{a_{2} \beta\left(1-z+j^{-1}\right)^{\beta-1}}{1-a_{1}^{2}}+\mathcal{O}\left(\left(1-z+j^{-1}\right)^{2 \beta-1}\right) \\
& -\frac{b_{2} \beta\left(1-z+j^{-1}\right)^{\beta-1}}{1-b_{1}^{2}}+\mathcal{O}\left(\left(1-z+j^{-1}\right)^{2 \beta-1}\right) \mid \\
\leq & \beta\left|\frac{a_{2}}{1-a_{1}^{2}}-\frac{b_{2}}{1-b_{1}^{2}}\right|\left(1-z+j^{-1}\right)^{\beta-1}+\mathcal{O}\left(\left(1-z+j^{-1}\right)^{2 \beta-1}\right) \\
= & \mathcal{O}\left(\left(1-z+j^{-1}\right)^{2 \beta-1}\right),
\end{aligned}
$$


where $\mathcal{O}\left(\left(1-z+j^{-1}\right)^{2 \beta-1}\right)$ denotes expressions which can be bounded by a constant (independent of $z$ or $j$ ) times $\left|1-z+j^{-1}\right|^{2 \beta-1}$. Since $2 \beta-1+\alpha>0$ by the assumption, it follows that the quantity $d_{\mathcal{B}_{\alpha}^{*}}\left(f_{j}, g_{j}\right)$ is uniformly bounded for all $j$.

\section{References}

[1] K. Deimling, Nonlinear functional analysis, Springer Verlag, Berlin, 1985.

[2] X. Li, On hyperbolic $Q$ classes, Dissertation, University of Joensuu, Joensuu, 2005, Ann. Acad. Sci. Fenn. Math. Diss. No. 145 (2005), 65 pp.

[3] X. Li, F. Pérez-González, and J. Rättyä, Composition operators in hyperbolic Q-classes, Ann. Acad. Sci. Fenn. Math. 31 (2006), 391-404.

[4] W. Smith, Inner functions in the hyperbolic little Bloch class, Michigan Math. J. 45 (1998), no. 1, 103-114.

[5] J. Xiao, Composition operators associated with Bloch-type spaces, Complex Variables Theory Appl. 46 (2001), no. 2, 109-121.

[6] J. Xiao, Holomorphic Q classes, Lecture Notes in Mathematics, 1767, SpringerVerlag, Berlin, 2001.

[7] S. Yamashita, Holomorphic functions of hyperbolically bounded mean oscillation, Boll. Un. Mat. Ital. B (6) 5 (1986), no. 3, 983-1000.

[8] S. Yamashita, Functions with $H^{p}$ hyperbolic derivative, Math. Scand. 53 (1983), no. 2, 238-244.

[9] S. Yamashita, Hyperbolic Hardy classes and hyperbolically Dirichlet-finite functions, Hokkaido Math. J. 10 (1981), Special Issue, 709-722.

[10] S. Yamashita, The hyperbolic M. Riesz theorem, Acta Sci. Math. (Szeged) 43 (1981), no. 1-2, 141-146.

[11] S. Yamashita, On hyperbolic Hardy classes, Comment. Math. Univ. St. Paul. 30 (1981), no. 1, 65-69.

[12] S. Yamashita, Hyperbolic Hardy class $H^{1}$, Math. Scand. 45 (1979), no. 2, 261266.

Fernando Pérez-González

Departamento de Análisis Matemático

Universidad de La Laguna

38271 La Laguna

Tenerife

Spain

e-mail: fernando.perez.gonzalez@ull.es

Jouni Rättyä

University of Joensuu

Department of Physics and Mathematics

P. O. Box 111

80101 Joensuu

Finland

e-mail: jouni.rattya@joensuu.fi 


\author{
Jari Taskinen \\ Department of Mathematics and Statistics \\ University of Helsinki \\ P.O. Box 68 \\ 00014 Helsinki \\ Finland \\ e-mail: jari.taskinen@helsinki.fi
}

